The Israeli Journal of Aquaculture - Bamidgeh, IJA_72.2020.1203799, 8 pages

http://doi.org/10.46989/IJA.72.2020.1203799

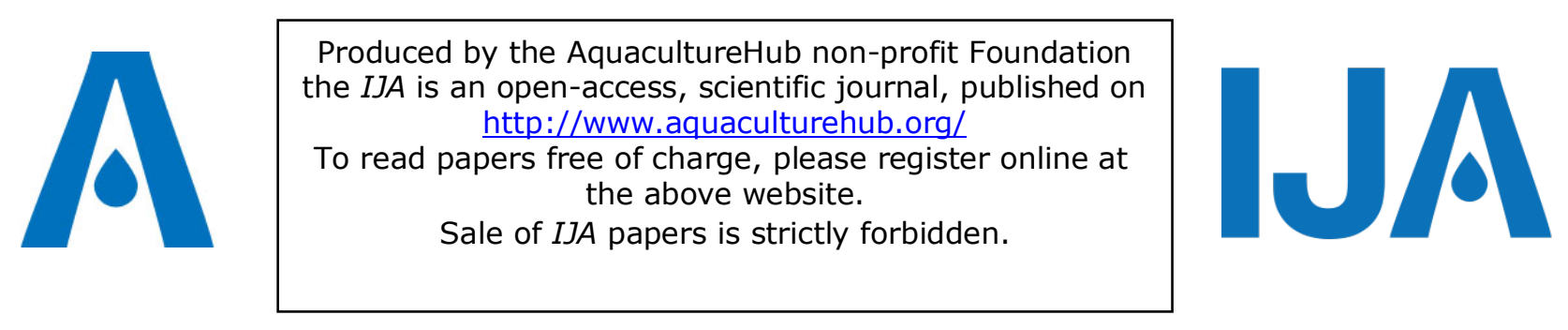

\title{
Research Progress on the Polymeric Immunoglobulin Receptor (pIgR) in Fish
}

\author{
Hu Xia ${ }^{1,2}$, Pinhong Yang ${ }^{*}$, Liangguo Liu ${ }^{1}$, Yushuang Luo ${ }^{1,2}$, Wenbin \\ Wang $^{1}$, Kezhong Cheng ${ }^{2}$, Liang $\mathrm{Ye}^{2}$, Dong Hou ${ }^{1}$, Jiemei Zhao ${ }^{1}$, Xian \\ $\mathrm{Wu}^{1}$
${ }^{1}$ Hunan Provincial Key Laboratory for Molecular Immunity Technology of Aquatic Animal Diseases, Hunan University of Arts and Science, Hunan Changde 415000

${ }^{2}$ Dahu Aquaculture company limited, Hunan Changde 415000

Key words: pIgR; Immunoglobulin; Mucosal immunity; Fish

\begin{abstract}
There are a large number of pathogens in the water where fish live, and the mucosal-associated lymphoid tissues (MALTs), such as skin, gill and intestine, are the first contact parts when pathogens infect fish. The secreted mucus of these tissues constitutes the first barrier for fish against the invasion of external pathogens. Mucosal immunity can identify and neutralize pathogens and induces immunocytes to devour pathogens and the like. As a key factor in the mucosal immune system, the polymeric immunoglobulin receptor (pIgR) is capable of mediating the transport and secretion of polymeric immunoglobulins towards mucus. The effective secretion of the pIgR is necessary for polymeric immunoglobulins (pIg) to exert mucosal defence and plays a significant role in fish immunity. With the deepening of research into fish immunoglobulins, the pIgR has become a research hotspot. The molecular structure, genetic structure and expression pattern of the pIgR and the important role it plays in mucosal immunity were summarized in this study, which contributed to a deeper understanding of fish mucosal immunity and laid a foundation for further exploration of the action mechanism and functions of the pIgR in fish.
\end{abstract}

* Corresponding author. e-mail: yph588@163.com 


\section{Introduction}

As an important constituent of the immunoglobulin (Ig) superfamily, the polymeric immunoglobulin receptor (pIgR) is synthesized by mucosal epithelial cells and exists in mucosal-associated lymphoid tissues (MALTs), such as stomach, intestinal and skin as well as in secretions. The pIgR can bind to secretory polymeric immunoglobulins (pIg) and mediate them to span epithelial cells for transport and secretion, thus ensuring that secretory Ig can locally scavenge pathogens and toxins in the mucosal defensive barrier (Gurevich et al., 2003; Kaetzel, 2005; Braathen et al., 2007). As first reported by Hamuro et al. (2007), just as the intestines of higher vertebrates, the skin of Takifugu rubripe transports immunoglobulin $\mathrm{M}$ (IgM) tetramer via the pIgR to mucous membranes (Hamuro et al., 2007). Since this realization, the important role played by the pIgR in fish mucosal immunity has gradually been brought to the forefront. In recent years, with the deepening of research on fish mucosal Ig, pIgR-mediated transport of mucosal Ig has attracted increasing attention. However, the genetic functions and action mechanisms of the pIgR are less understood. The progress of the research on the structure and functions of the pIgR in fish is hereby reviewed, expecting to guide future research on the functions and action mechanisms of the pIgR in mucosal immunity of fish.

\section{Molecular structure of a pIgR}

The pIgR is an important immune molecule that assists in the transport of antibody molecules from the basilar membrane to the apical membrane of epithelial cells for mucosal immunity (Kaetzel, 2005); it is also a Type I transmembrane glycoprotein. The gene sequence of pIgR was cloned from a mammal (a rabbit) in 1985 (Deitcher and Mostov, 1986); the pIgR gene was then cloned from the gene sequences of higher vertebrates such as Homo sapiens, Mus musculus, Bos taurus and Gallus gallus (Hempen et al., 2002; Piskurich et al., 1995; Verbeet et al., 1995; Fodor et al., 1997). Subsequently, this gene was cloned from Ornithorhynchus anatinus and Xenopus laevis (Braathen et al., 2007). The pIgR gene sequence has also been cloned from fish genes in recent years. Mammalian pIgR proteins have a common structural feature: All are Type I transmembrane proteins with a molecular weight of about $100 \mathrm{kDa}$ (Kaetzel, 2005). Their structures are divided into one extracellular region, one transmembrane region and one intracellular region, where the extracellular region contains five Ig functional domains, which are similar to a variable Ig structure to a great extent (Krajci et al., 1989; Mostov et al., 1984). Therefore, the five functional domains are called Ig-like domains (ILDs), which can be regarded as a standard structural model of pIgR, as shown in Figure 1. (Mostov et al., 1984). In mammals, ILD1, ILD4 and ILD5 are highly conservative, and ILD1 is a binding site of immunoglobulin A (IgA). Research reports show that after ILD1 mutation is realized through a point mutation method in a rabbit, the pIgR will fail to bind to IgA, proving that ILD1 is an indispensable structure for Ig binding (Kaetzel, 2005). There are five ILD structural domains in mammalian pIgR, but the quantity of ILD structural domains varies in other animals. The pIgR contains three ILD structural domains in rabbits and cows corresponding to ILD1, ILD4 and ILD5 in Homo sapiens, respectively (Mostov et al., 1980; Kühn et al., 1981; Kulseth et al., 1995). Extracellular regions in birds and amphibians contain four ILD structural domains, where the ILD structural domains in birds correspond to ILD1 and ILD3-5 of mammals (Wieland et al., 2004). In Teleost, the pIgR gene was firstly cloned from Takifugu rubripes, which was proved to contain only two ILD structural domains. Subsequently, this gene was cloned from Cyprinus carpio, Paralichthys olivaceus, Epinephelus coioides, among others. According to related analyses, these fish also contained two ILD structural domains (Xu et al., 1980; Rombout et al., 2008; Hamuro et al., 2007; Feng et al., 2009). Bioinformatic analysis reveals that the first ILD structural domain corresponds to the ILD1 domain in fish and that the second ILD domain corresponds to the ILD5 domain in mammals. It is widely accepted that the second ILD domain in fish is ILD5. Although the two ILDs are in series connection, they do not exist in the form of spliceosome (Xu et al., 1980; Rombout et al., 2008; Hamuro et al., 2007; Feng et al., 2009). 


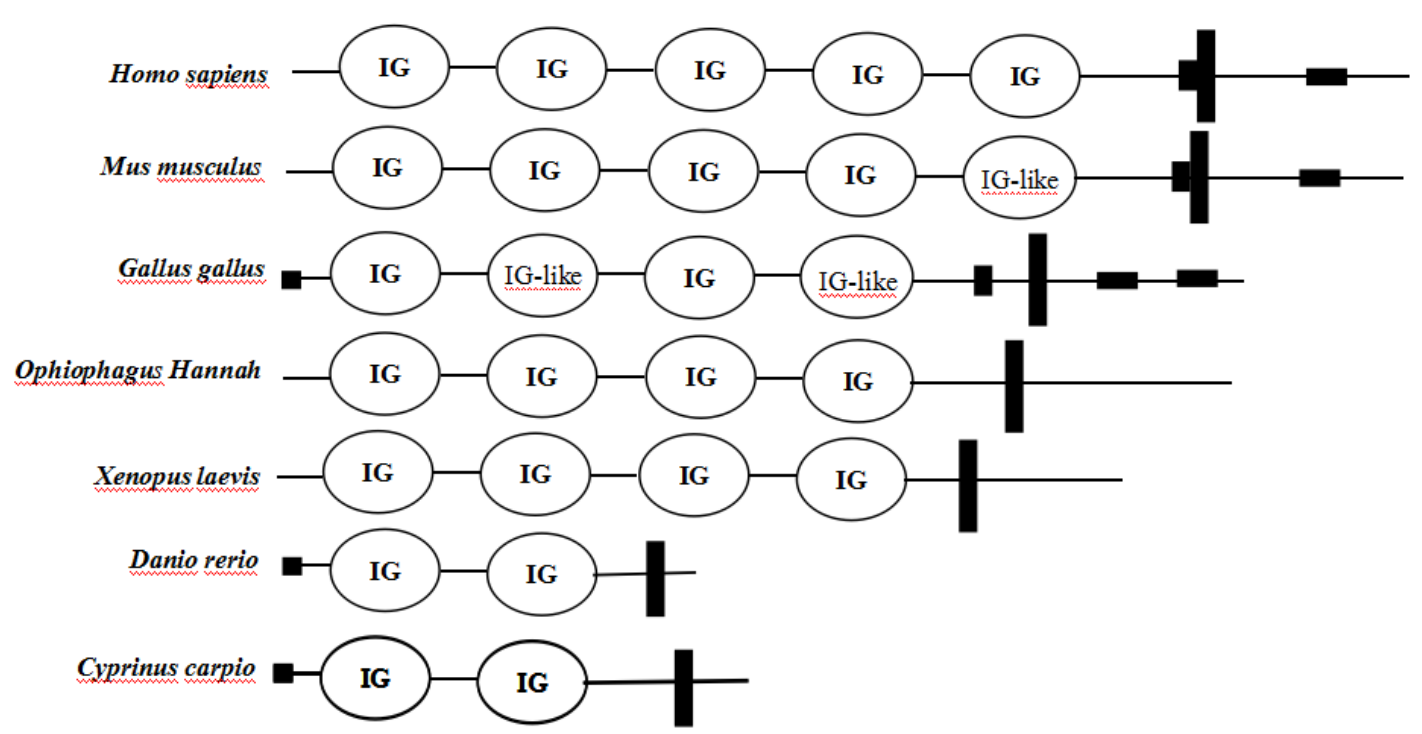

Figure 1 The pIgR structure profile of Homo sapiens; Mus musculus; Gallus gallus; Ophiophagus Hannah; Xenopus laevis; Danio rerio; Cyprinus carpio.

The studies in mammals show that the extracellular ligand-binding domain of the pIgR is a kind of glycoprotein, which was initially called 'secretory piece' but is currently known as a 'secretory component' (SC). SC is not generated by plasmocyte and its molecular weight is $80 \mathrm{kD}$ or so; soluble SC is hydrolytic fragment of the pIgR (Hempen et al., 2002; Mostov et al., 1980). In Homo sapiens, the pIgR is a glycoprotein, and research indicates that there are seven glycosylation sites on the surface of the pIgR in Homo sapiens (Hughes et al., 1999; Eiiert et al., 1991). However, there is some controversy about the N-glycosylation site of pIgR. Glycosylated SC allows better antibody secretion into body fluids (Scheiffele and Fuilekrug, 2000; Daly et al., 2007). However, other studies suggest that the glycosylation of recombined SC is not necessary for the binding of a pIgR to an antibody (Prinsloo et al., 2006). Fish have few or no pIgR glycosylation sites: Cyprinus carpio and Takifugu rubripes each have one glycosylation site while Epinephelus coioides does not, indicating that glycosylation has no bearing on the binding of a pIgR to an antibody (Rombout et al., 2008; Hamuro et al., 2007; Feng et al., 2009).

\section{The genetic structure of the pIgR}

The human pIgR gene is located on chromosome 1 and contains 11 exons and 10 introns with a length of $17944 \mathrm{bp}$ (Martin et al., 1998). The pIgR genes of both rats and rabbits are composed of 11 exons and 10 introns (Martin et al., 1997). Unlike the pIgR gene of Homo sapiens, the pIgR gene of Epinephelus coioides consists of 8 exons and 7 introns; that of Takifugu rubripes contains 8 exons and 7 introns with a full length of 2.8 $\mathrm{kbp}$; that of Danio rerio is located on chromosome 2 and consists of 8 exons and 7 introns. Sequence alignment shows that the DAD1L gene and the LRRC24L gene are located at two sides of the pIgR gene of a Brachydanio rerio chromosome, and that they also exist in the genomes of Takifugu rubripes, Oreochromis mossambicus and Oryzias latipes. However, the three genes exist on three different chromosomes of the Homo sapiens genome. It is speculated that this arrangement can be attributed to genome doubling or missing during the evolution process (Kortum et al., 2014).

\section{The expression pattern of the pIgR}

Mammalian pIgR is expressed in both mucosal tissues (skin, intestine, respiratory tract and stomach) and lymphoid tissues (spleen, thymus and head kidney), and is 
especially prevalent in mucosal immunity-associated tissues. It mainly exists in epithelial cells inside mucosal-related tissues and on the basilar membrane of multiple glandular epithelial cells. Based on the analysis of differential expressions in tissues of Teleost, the pIgR gene is expressed in tissues of Takifugu rubripes (Hamuro et al., 2007), Epinephelus coioides (Feng et al., 2009), Cyprinus carpio, L. (Rombout et al., 2008), Paralichthys olivaceus (Xu et al., 2013) and Scophthalmus maximus (Ding et al., 2013), and its expression is usually high in mucus-associated tissues, such as skin, gill, intestinal and stomach. Hamuro et al. (2007) investigated tissue localization of the pIgR using in situ hybridization (ISH) and found that the pIgR gene presented a positive signal in the epithelial cells of skin and the intestines of Takifugu rubripes (Hamuro et al., 2007). Similarly, by using ISH, Rombout et al. (2008) found that mRNAs of a Cyprinus carpio pIgR gene were distributed among epithelial cells in mucosal-associated tissues, such as intestinal and skin (Rombout et al., 2008). Ding et al. (2013) conducted tissue localization of Scophthalmus maximus pIgRs via immunohistochemical staining and found that the pIgR gene showed red positive signals in the epithelial cells of four tissues: skin, gill, stomach and intestinal(Ding et al., 2013). These studies show that the distribution of pIgRs corresponds to the function of binding and transporting pIg.

Rabot et al. (2013) pointed out that with stimulation by peptidoglycan (LPS), the expression quantity of the pIgR gene first increased and then declined in bovine mammary epithelial cells (Rabot et al., 2013). In teleost, Yu et al. (2018) detected the infection of Misgurnus anguillicaudatus by Aeromonas hydrophila and Ichthyophthiriasis using real-time fluorescence quantitative PCR (QRT-PCR), and found that pIgR expression tended first to increase and then decline in the kidney, spleen, intestines and gills (Yu et al., 2018). Stimulated by a solution of inactivated Vibrio anguillarum, the relative pIgR expressions in tissues, such as skin, intestinal, gill, stomach, head kidney and spleen, of Scophthalmus maximus all tended clearly first to increase and then to decrease, indicating that in the initial phase of defence against infection, the expression regulation of the pIgR gene in teleost was influenced by external immunostimulation and that the pIgR participated in a mucosal immune response (Ding et al., 2013). Meanwhile, semi-quantitative RT-PCR results showed that pIgR was distributed in all tissues in healthy Cyprinus carpio and Scophthalmus maximus, indicating that pIgR gene presents a combinatorial and inducible co-existing expression pattern in Cyprinus carpio and Scophthalmus maximus (Rombout et al., 2008 ; Ding et al., 2013).

\section{Functions of the pIgR}

As the first immune-defence barrier, the mucosal immune system can participate in protecting living organisms from being infected by pathogens. The $\mathrm{pIgR}$, which is a key factor in mucosal immune defence, is capable of mediating pIg to span epithelial cells for transport and secretion so as to locally scavenge pathogens and toxins in the mucosal barrier. Hence, effective secretion of pIgRs is a requirement for mucosal Ig in successful immune defence (Gurevich et al., 2003; Kaetzel et al., 2005; Braathen et al., 2007). Among mammals, pIgRs in Homo sapiens can not only transport IgA polymers but also IgM polymers, while the pIgRs of other species (e.g. rabbits, rodents, chickens) transport only IgA (Underdown et al., 1992; Wieland et al., 2004). After being synthesized on endoplasmic reticulum, the pIgR can bind to pIg on the basal surface of epithelial cells to form the compound pIgR-pIg, which is ingested by epithelial cells through endocytosis; the pIgR-pIg is then transported to the apical surface of cells and hydrolyzed by protease. Further, the SC of a pIgR can bind to pIg to form secretory Ig (Pilette, 2003; Gurevich et al., 2003; Brandtzaeg, 2003). The binding of SC to pIg can protect pIg in mucus from being degraded by proteolytic enzymes, meanwhile strengthening the stability of pIg in mucus, thus ensuring that secretory Ig can give full play to its function of immune defence in mucus (Chintalacharuvu and Morrison, 1997; Crottet and Corthesy, 1998); pIgR that does not bind to Ig can return to the basal surface of epithelial cells again and continue to mediate Ig transport in the same way (Goldman, 1993). In teleost, IgM in skin and intestinal mucus is an important molecule in mucosal immune system. Research on Takifugu rubripes shows that the skin of teleost and the 
intestines of mammals share the same Ig transport system: tetramer IgM is transported via pIgRs in epithelial cells and then enters mucus to exert its immunological function (Hamuro et al., 2007). The function of pIgRs in mucosal immunity of teleost is then of increased interest. Studies show that there are multiple mucosal Igs in teleost. Where IgM participates in skin mucosal immunity, IgM and IgZ/IgT participate in intestinal mucosal immunity; IgM and IgT can enter mucus only by binding to a pIgR, which is similar to the transport of IgA and IgM in mammals (Underdown et al., 1992; Wieland et al., 2004; Zhang et al., 2010). This fully certifies that pIgR plays a part in the transport of pIg towards mucus in teleost and is the precondition for mucus to function in immune defence.

Besides mediating and transporting Ig into mucus, the pIgR also has the function of nonspecific immune defence. To be specific, the pIgR can stimulate the synthesis of other immune factors; after a pIgR is hydrolyzed by protease, the dissociative SC can prevent chemotaxis of neutrophilic granulocytes so as to relieve the inflammatory response of an organism and protect epithelial cells; furthermore, it can bind to bacteria to effectively restrict bacterial infection in an organism; in addition, SC can resist the degradation of neutrophil elastase and further enhance humoral immunity of the respiratory tract in humans (Pilette et al., 2003). Therefore, the pIgR exerts an immune defence function in living organisms and plays a critical role in mucosal immune defence. Therefore, the further exploration of the structure and functions of the pIgR can provide more theoretical bases and new ideas for intensive research of the biological immune system.

\section{Conclusion}

Research into fish mucosal immunity has made enormous progress in recent years. As new types of mucosal Igs, such as IgT, are examined closely, the research into fish mucosal Ig is entering a new era. The pIgR has a decisive effect in fish mucosal immunity, and its functions in mucosal antibody secretion and immune defence have become a research hotspot in the field of mucosal immunity. Although progress has been achieved in research with regard to the pIgR of fish - including structure, function and expression regulation - various problems, such as transcytosis of the pIgR on Ig, the Ig transport path and mechanisms that regulate expression, remain unclear and in need of further investigation. Research into the structure and biological functions of the pIgR will deepen the understanding of mucosal immune systems in fish, lay a foundation for developing a new vaccine that can induce serum antibodies and mucosal antibodies simultaneously and boost the development of new fish vaccination strategies.

\section{Acknowledgements}

The present study was supported by the Hunan Natural Science Foundation (Grant No. 2018JJ3373), China Postdoctoral Science Foundation (Grant No. 2019M652748), Hunan Provincial Education Department Outstanding Youth Fund (Grant No. 19B382), Chang Cai Qi Zhi (2019) No.71, Hunan university of arts and science 2018 University Students Innovation and Entrepreneurship Research Project (ZD1802), Hunan Provincial Key Laboratory for Microbial Molecular Biology (Grant No. 2019FW05) and Co-construction of Provinces and Ministries State Key Experiment on Developmental Biology of Freshwater Fishes Open-end Fund project (Grant No.2017KF007). The authors declare no conflict of interests.

\section{References \\ Braathen R., Hohman V. S., Brandtzaeg P., Johansen F. E., 2007. Secretory antibody formation: conserved binding interactions between J chain and polymeric Ig receptor from humans and amphibians. Journal of immunology, 178(3): 1589-1597. doi:10.4049/jimmunol.178.3.1589.}

Chintalacharuvu K. R., Morrison S. L., 1997. Production of secretory immunoglobulin A by a single mammalian cell. Proceedings of the National Academy of Sciences of the United States of America, 94(12): 6364-6368. DOI:10.1073/pnas.94.12.6364. 
Crottet P., Corthésy B., 1998. Secretory component delays the conversion of secretory IgA into antigen-binding competent $F\left(a b^{\prime}\right) 2$ : a possible implication for mucosal defense. Journal of immunology, 161(10): 5445-5453. PMID: 9820520.

Daly K. A., Digby M., Lefèvre C., Mailer S., Thomson P., Nicholas K., Williamson P., 2007. Analysis of the expression of immunoglobulins throughout lactation suggests two periods of immune transfer in the tammar wallaby (Macropus eugenii). Veterinary immunology and immunopathology, 120(3-4): 187-200. doi:10.1016/j.vetimm.2007.07.008.

Ding B. J., Sheng X. Z., Tang X. Q., Xing J., Zhan W.B., 2013. Molecular cloning and expression analysis of the pIgR gene in Scophthalmus maximus. Journal of Fishery Sciences of China, 20(4): 792-801. doi:10.1016/j.fsi.2013.05.024 .

Deitcher D. L, Mostov K. E., 1986. Alternate splicing of rabbit polymeric immunoglobulin receptor. Molecular Cellular Biology, 6(7): 2712-2715. doi:10.1128/mcb.6.7.2712.

Eiffert H., Quentin E., Wiederhold M., Hillemeir S., Decker J., Weber M., Hilschmann N., 1991. Determination of the molecular structure of the human free secretory component. Biological chemistry Hoppe-Seyler, 372(2): 119-128. doi:10.1515/bchm3.1991.372.1.119.

Feng L. N., Lu D. Q., Bei J. X., Chen J. L., Liu Y., Zhang Y., Liu X. C., Meng Z. N., Wang L., Lin H. R., 2009. Molecular cloning and functional analysis of polymeric immunoglobulin receptor gene in orange-spotted grouper (Epinephelus coioides). Comparative biochemistry and physiology. Part B, Biochemistry \& molecular biology, 154(3): 282-289. doi:10.1016/j.cbpb.2009.07.003.

Fodor E., Feren A., Jones, A., 1997. Isolation and genomic analysis of the rat polymeric immunoglobulin receptor gene terminal domain and transcriptional control region. DNA and cell biology, 16(2): 215-225. doi:10.1089/dna.1997.16.215.

Goldman A. S., 1993. The immune system of human milk: antimicrobial, antiinflammatory and immunomodulating properties. The Pediatric infectious disease journal, 12(8): 664-671. doi:10.1097/00006454-199308000-00008.

Gurevich P., Zusman I., Moldavsky M., Szvalb S., Elhayany A., Halperin R., Gurevich E., Ben-Hur H., 2003. Secretory immune system in human intrauterine development: immunopathomorphological analysis of the role of secretory component (pIgR/SC) in immunoglobulin transport (review). International journal of molecular medicine, 12(3): 289-297. PMID: 12883643.

Hamuro K., Suetake H., Saha N. R., Kikuchi K., Suzuki Y., 2007. A teleost polymeric Ig receptor exhibiting two Ig-like domains transports tetrameric IgM into the skin. Journal of immunology, 178(9): 5682-5689. doi:10.4049/jimmunol.178.9.5682.

Hempen P. M., Phillips K. M., Conway P. S., Sandoval K. H., Schneeman T. A., Wu H. J., Kaetzel C. S., 2002. Transcriptional regulation of the human polymeric Ig receptor gene: analysis of basal promoter elements. Journal of immunology, 169(4): 1912-1921. doi:10.4049/jimmunol.169.4.1912.

Hughes G. J., Reason A. J., Savoy L., Jaton J., Frutiger-Hughes S., 1999. Carbohydrate moieties in human secretory component. Biochimica et biophysica acta, 1434(1): 86-93. doi:10.1016/s0167-4838(99)00168-5.

Kaetzel C. S., 2005. The polymeric immunoglobulin receptor: bridging innate and adaptive immune responses at mucosal surfaces. Immunological reviews, 206: 83-99. doi:10.1111/j.0105-2896.2005.00278.x.

Krajci P., Solberg R., Sandberg M., Oyen O., Jahnsen T., Brandtzaeg, P., 1989. Molecular cloning of the human transmembrane secretory component (poly-Ig receptor) and its mRNA expression in human tissues. Biochemical and biophysical research communications, 158(3): 783-789. doi:10.1016/0006-291x(89)92790-3.

Kortum A. N., Rodriguez-Nunez I., Yang J., Shim J., Runft D., O'Driscoll M. L., Haire R. N., Cannon J. P., Turner P. M., Litman R. T., Kim C. H., Neely M. N., Litman G. W., Yoder J. A., 2014. Differential expression and ligand binding indicate alternative functions for zebrafish polymeric immunoglobulin receptor (pIgR) and a family 
of pIgR-like (PIGRL) proteins. Immunogenetics, 66(4): 267-279. doi:10.1007/s00251014-0759-4.

Kühn L. C., Kraehenbuhl J.P., 1981. The membrane receptor for polymeric immunoglobulin is structurally related to secretory component. Isolation and characterization of membrane secretory component from rabbit liver and mammary gland. Journal of Biological Chemistry, 256(23): 2490-2495. PMID: 7298670.

Kulseth M. A., Krajci P., Myklebost O., Rogne S., 1995. Cloning and characterization of two forms of bovine polymeric immunoglobulin receptor CDNA. DNA and cell biology, 14(3): 251-256. doi:10.1089/dna.1995.14.251.

Martin M. G., Wang J.,Li T. W., 1998. Characterization of the 5 '-flanking region of the murine polymeric Ig A receptor gene. Am J Physiol, 275(4 Pt 1): G778-88. doi:10.1152/ajpgi.00420.2001.

Martín M. G., Gutierrez E. M., Lam J. T., Li T. W., Wang J., 1997. Genomic cloning and structural analysis of the murine polymeric receptor (pIgR) gene and promoter region. Gene, 201(1-2): 189-197. doi:10.1016/s0378-1119(97)00447-2.

Mostov K. E., Friedlander M., Blobel G., 1984. The receptor for transepithelial transport of IgA and IgM contains multiple immunoglobulin-like domains. Nature, 308(5954): 37-43. doi:10.1038/308037a0.

Mostov K. E., Kraehenbuhl J. P., Blobel G., 1980. Receptor-mediated transcellular transport of immunoglobulin: synthesis of secretory component as multiple and larger trans-membrane forms. Proceedings of the National Academy of Sciences of the United States of America, 77: 7257-7261. doi:10.1073/pnas.77.12.7257.

Pilette C., Ouadrhiri Y., Dimanche F., Vaerman J. P., Sibille Y., 2003. Secretory component is cleaved by neutrophil serine proteinases but its epithelial production is increased by neutrophils through NF-kappa B- and p38 mitogen-activated protein kinasedependent mechanisms. American journal of respiratory cell and molecular biology, 28(4): 485-498. doi: $10.1165 / \mathrm{rcmb} .4913$.

Piskurich J. F., Blanchard M. H., Youngman K. R., France J. A., Kaetzel, C. S., 1995. Molecular cloning of the mouse polymeric Ig receptor. Functional regions of the molecule are conserved among five mammalian species. Journal of immunology, 154(4): 1735-1747. PMID: 7836758.

Prinsloo E., Oosthuizen V., Muramoto K., Naude R. J., 2006. In vitro refolding of recombinant human free secretory component using equilibrium gradient dialysis. Protein expression and purification, 47(1): 179-185. doi:10.1016/j.pep.2005.09.017.

Rabot A., Wellnitz O., Meyer H. H. D., Bruckmaier R. M., 2007. Use and relevance of a bovine mammary gland explant model to study infection responses in bovine mammary tissue. Journal of Dairy Research, 74(1): 93-99. doi: $10.1017 /$ S0022029906002147.

Rombout J. H., van der Tuin S. J., Yang G., Schopman N., Mroczek A., Hermsen T., Taverne-Thiele J. J., 2008. Expression of the polymeric Immunoglobulin Receptor (pIgR) in mucosal tissues of common carp (Cyprinus carpio L.). Fish \& shellfish immunology, 24(5): 620-628. doi:10.1016/j.fsi.2008.01.016.

Scheiffele P., Füllekrug J., 2000. Glycosylation and protein transport. Essays in biochemistry, 36: 27-35. doi:10.1042/bse0360027.

Underdown B. J., Switzer I., Jackson G. D., 1992. Rat secretory component binds poorly to rodent IgM. Journal of immunology, 149(2): 487-491. PMID: 1624795.

Verbeet M. P., Vermeer H., Warmerdam G. C., de Boer H. A., Lee S. H., 1995. Cloning and characterization of the bovine polymeric immunoglobulin receptor-encoding cDNA. Gene, 164(2): 329-333. doi:10.1016/0378-1119(95)00520-g.

Wieland W. H., Orzáez D., Lammers A., Parmentier H. K., Verstegen M. W., Schots A., 2004. A functional polymeric immunoglobulin receptor in chicken (Gallus gallus) indicates ancient role of secretory IgA in mucosal immunity. The Biochemical journal, 380(Pt 3): 669-676. doi:10.1042/BJ20040200.

Xu G., Zhan W., Ding B., Sheng X., 2013. Molecular cloning and expression analysis of polymeric immunoglobulin receptor in flounder (Paralichthys olivaceus). Fish \& shellfish immunology, 35(3): 653-660. doi:10.1016/j.fsi.2013.05.024. 
Yu Y., Liu Y., Li H., Dong S., Wang Q., Huang Z., Kong W., Zhang X., Xu Y., Chen X., Xu, Z., 2018. Polymeric immunoglobulin receptor in dojo loach (Misgurnus anguillicaudatus): Molecular characterization and expression analysis in response to bacterial and parasitic challenge. Fish \& shellfish immunology, 73: 175-184. doi:10.1016/j.fsi.2017.12.019.

Zhang Y. A., Salinas I., Li J., Parra D., Bjork S., Xu Z., LaPatra S. E., Bartholomew J., Sunyer J. O., 2010. IgT, a primitive immunoglobulin class specialized in mucosal immunity. Nature immunology, 11(9): 827-835. doi:10.1038/ni.1913. 